\title{
COSTO-EFECTIVIDAD DEL DIAGNÓSTICO ETIOLÓGICO DE LA CERVICITIS POR C. TRACHOMATIS BASADO EN PRUEBAS RÁPIDAS COMPARADO CON EL ABORDAJE SINDRÓMICO, EN MUJERES NO GESTANTES CON SÍNTOMAS DE INFECCIÓN DEL TRACTO GENITAL INFERIOR
}

\section{Cost-effectiveness of etiologic diagnosis for C. trachomatis infection based on rapid tests versus syndromic approach in non-pregnant women with symptoms of lower genital tract infection}

Víctor Prieto-Martínez, Econ ${ }^{1}$; Alexander Moreno-Calderón, $M S c^{1}$; José Urrego-Novoa, $M S c^{1}$; Jorge Augusto Díaz-Rojas, MSc $^{1}$; Andrea Rodríguez-Hernández, MD, MSc ${ }^{1}$; Ariel Iván Ruiz-Parra, MD, $\mathrm{MSc}^{1,2}$; Hernando Gaitán-Duarte MD, $\mathrm{MSc}^{1,2}$; Jorge E. Tolosa-Ardila, MD, $\mathrm{MSc}^{3}$

Recibido: Enero 24/14 - Aceptado: Diciembre 15/14

\section{RESUMEN}

Objetivo: estimar la razón costo-efectividad del diagnóstico etiológico con pruebas rápidas para la cervicitis por $C$. trachomatis frente al diagnóstico sindrómico, en mujeres no gestantes con síntomas de infección del tracto genital inferior en Colombia. Materiales y métodos: se construyó un árbol de decisión para determinar la razón de costo-efectividad de la aproximación etiológica con las pruebas rápidas Acon ${ }$ Plate, Acon ${ }^{\circledR}$ Duo y QuickVue ${ }^{\circledR}$

1 Instituto de Investigaciones Clínicas, Universidad Nacional de Colombia, Bogotá (Colombia).vaprietom@gmail.com

2 Departamento de Obstetricia y Ginecología, Universidad Nacional de Colombia, Bogotá (Colombia).

3 Department of Obstetrics \& Gynecology, Oregon Health \& Science University. Global Network for Perinatal \& Reproductive Health, Portland, Oregon (USA). para la detección de C. trachomatis comparada con el diagnóstico sindrómico. La perspectiva fue la del sistema de salud colombiano incluyendo todos los costos médicos directos. El horizonte de tiempo fue de 15 días, ya que la unidad de resultado fue el número de casos correctamente identificados (número de verdaderos positivos y verdaderos negativos). Las características operativas de las pruebas se obtuvieron en un estudio de corte transversal diseñado y conducido para este propósito.

Resultados: la alternativa más costosa y más efectiva fue QuickVue® seguida de Acon®Plate y del abordaje sindrómico. Acon ${ }$ Duo fue una estrategia dominada. La razón de costo-efectividad incremental de QuickVue ${ }^{\circledR}$, comparada con Acon $®$ Plate, fue de \$ 430.671; la de Acon®Plate, comparada con el abordaje sindrómico, fue de $\$ 79.747$. 
Conclusión: si la disponibilidad a pagar (DAP) por un caso correctamente identificado adicional es mayor que \$ 430.671, QuickVue ${ }^{\circledR}$ sería la mejor alternativa en términos de costo-efectividad; de otro lado, si la DAP está entre \$ 79.747 y \$ 430.671, Acon ${ }^{\circledR P l a t e}$ sería la alternativa costo-efectiva. Finalmente, si la DAP es menor que $\$ 79.747$, el abordaje sindrómico sería la mejor alternativa en términos de costo-efectividad.

Palabras clave: análisis costo-beneficio, cervicitis, Colombia.

\section{ABSTRACT}

Objective: To estimate the cost-effectiveness of etiological approach with rapid tests for $C$. trachomatis cervicitis versus syndromic diagnosis, in non-pregnant women with symptoms of lower genital tract infection in Colombia.

Materials and methods: A decision tree was developed for determining the cost-effectiveness ratio of the aetiological approach using the Acon ${ }^{\circledR P l a t e}$, Acon ${ }^{\circledR}$ Duo and QuickVue ${ }^{\circledR}$ quick tests for the detection of $C$. trachomatis, compared with the syndromic diagnosis. The perspective was that of the Colombian healthcare system, including medical direct costs. The time period was 15 days, considering that the outcome unit was the number of cases identified correctly (number of true positives and true negatives). The operational characteristics of the tests were derived from a cross-sectional study designed and conducted for that specific purpose.

Results: The more costly and effective option was QuickVue $\AA$, followed by Acon $®$ Plate and the syndromic approach. Acon $®$ Duo was a dominated strategy. The incremental cost-effectiveness ratio for QuickVue ${ }^{\circledR}$ compared with Acon®Plate was \$430.671, and that of Acon®Plate compared with the syndromic approach was $\$ 79.747$.

Conclusion: If the willingness to pay (WTP) for an additional case that is correctly identified is greater than $\$ 430.671$, QuickVue ${ }^{\circledR}$ would be the best option in cost-effectiveness terms. On the other hand, if the WTP is between $\$ 79.747$ and $\$ 430.671$, Acon $囚$ Plate would be the cost-effective strategy. Finally, if the WTP is less than $\$ 79.747$, the syndromic approach would be the best option in cost-effectiveness terms.

Key words: Cost-benefit analysis, cervicitis, Colombia.

\section{INTRODUCCIÓN}

Las infecciones de transmisión sexual (ITS) continúan siendo un problema de salud pública de gran importancia en todas las regiones del mundo. Se estima que cada año ocurren 448 millones de casos nuevos de ITS en adultos de 15 a 49 años de edad. Estas son causadas especialmente por sífilis, gonorrea, clamidia y trichomonas, sin incluir casos de virus de inmunodeficiencia humana (VIH) u otras ITS (1). Las ITS generan una importante carga de enfermedad ya que pueden causar enfermedad pélvica inflamatoria con la subsecuente infertilidad y dolor pélvico crónico en el caso de la clamidia y la gonorrea $(2,3)$, o malformaciones congénitas en el caso de la sífilis gestacional (4).

Para el manejo de las ITS se ha propuesto el abordaje sindrómico, bajo el supuesto de que los diferentes síndromes (descarga uretral, cervicitis, flujo vaginal, úlcera genital, etc.) son causados por gérmenes específicos que pueden ser tratados por esquemas antibióticos definidos en el momento de realizar el diagnóstico. Este abordaje es sugerido por la Organización Mundial de la Salud (OMS) para países de bajos o medianos ingresos, donde no hay disponibilidad de laboratorios y las pruebas etiológicas (cultivos o pruebas de amplificación de ácidos nucleicos - NAAT), además de costosas, requieren una segunda consulta para establecer el tratamiento, con el riesgo de que la paciente no regrese y se pierda la oportunidad de tratarla (5). En Colombia, el Ministerio de Salud y Protección Social recomendó el abordaje sindrómico para el tratamiento de las ITS (6).

Respecto al síndrome de cervicitis, este es causado principalmente por la $N$. gonorroheae y la 
C. trachomatis (7). Dado que las infecciones por $C$. trachomatis son en gran proporción asintomáticas (8), se ha sugerido que en la cervicitis el abordaje sindrómico podría no ser la mejor opción de manejo (9).

Como alternativa al abordaje sindrómico se ha propuesto el uso de pruebas rápidas para $C$. trachomatis y $N$. gonorroheae aplicadas en el sitio de atención, en virtud de que dichas pruebas tendrían una mejor exactitud diagnóstica y permitirían documentar la presencia de cervicitis al momento de la consulta de manera que se pueda dar tratamiento específico inmediato, lo que representaría menos casos de infección sin tratamiento o sobretratamiento (10). No obstante, la aplicación de esta tecnología implicaría un mayor costo cuando se compara con el abordaje sindrómico. Vale la pena anotar que en el sistema de salud colombiano las pruebas rápidas no están incluidas en el Plan Obligatorio de Salud (POS) (11).

El objetivo de este estudio es estimar la razón de costo-efectividad del diagnóstico etiológico basado en pruebas rápidas para cervicitis por $C$. trachomatis, comparándolo con el abordaje sindrómico en mujeres no gestantes con síntomas sugestivos de infección del tracto genital inferior.

\section{MATERIALES Y MÉTODOS}

Evaluación económica ensamblada sobre un estudio de prevalencia de ITS en mujeres de 14 a 49 años, sexualmente activas, con síntomas sugestivos de infección del tracto genital, que mostró una prevalencia de 9,7\% (IC 95\%: 8,2-11,4) para la C. trachomatis (12), y que además evaluó la exactitud del manejo sindrómico (13) y de las pruebas rápidas para C. trachomatis y N. gonorroheae (14).

La evaluación económica compara los costos y la efectividad (en términos de las características operati-

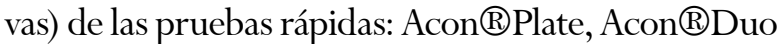
y QuickVue ${ }^{\circledR}$ con el abordaje sindrómico, para el diagnóstico de cervicitis por $C$. trachomatis. Este estudio se realizó desde la perspectiva del sistema de salud colombiano, incluyendo solo los costos médicos directos (15). Se empleó un horizonte temporal de dos semanas debido a que solo se tomó en cuenta el desempeño operativo de la prueba diagnóstica (pruebas rápidas o diagnóstico sindrómico) en términos de su capacidad para diagnosticar a quienes tienen o no tienen la infección y, de esta manera, recibir el tratamiento pertinente. No se incluyeron desenlaces a mediano y largo plazo -como enfermedad pélvica inflamatoria o secuelas como infertilidad- ya que para esta evaluación se debería incluir la efectividad del tratamiento, lo que está por fuera del alcance de esta publicación. Este periodo de dos semanas tomó en cuenta el tiempo durante el cual se realizan las pruebas rápidas, se desarrolla la prueba patrón de oro y se entregan y discuten los resultados de laboratorio con las participantes. Dado que el horizonte temporal es inferior a un año no se aplicó descuento sobre los costos y resultados en salud.

Medida de efectividad. Los resultados en salud se midieron en unidades de desempeño operativo de las dos alternativas diagnósticas evaluadas: casos correctamente identificados, definidos como la proporción de verdaderos positivos y verdaderos negativos, a partir de sus características operativas (sensibilidad y especificidad).

Uso de recursos y costos. Para estimar el consumo de recursos se realizó un consenso de expertos que construyó el caso tipo, esto es, un caso representativo de las mujeres no gestantes con síntomas de cervicitis por C. trachomatis, siguiendo la metodología Bottom Up (16). Todas las unidades monetarias se expresaron en pesos colombianos de 2010. La fuente de costos para los procedimientos fue el Manual de Tarifas de Instituto de Seguros Sociales (ISS) año 2001 (17), con un incremento del 30\% (caso base) que obedece al comportamiento en negociaciones con prestadores en el sistema de salud colombiano, cifra que para el análisis de sensibilidad tomó valores incrementales de 25 a $48 \%$. El caso base y el rango de variación fueron identificados a partir de una encuesta que se realizó a varios prestadores para el desarrollo de la "Guía de práctica clínica para el manejo sindrómico de las ITS" (6).

El costo de las pruebas rápidas fue tomado de la cotización con los proveedores, y para los análisis 
de sensibilidad se asumió un rango de $30 \%$ sobre el costo total de las pruebas, en relación con el comportamiento del mercado observado durante el año. En la tabla 1 se presentan los costos utilizados en la evaluación económica.

Datos epidemiológicos y características operativas. Se utilizó la sensibilidad y especificidad de cada una de las alternativas diagnósticas y la prevalencia global de la cervicitis por $C$. trachomatis (12). Adicionalmente, para el cálculo de los límites mínimo y máximo se utilizó la fórmula de la estimación del intervalo de confianza (IC $95 \%$ ) para una proporción, como se muestra a continuación:

$\hat{p}-1,96 * \sqrt{\frac{\hat{p}(1-\hat{p})}{n}} \leq p \leq \hat{p}+1.96 * \sqrt{\frac{\hat{p}(1-\hat{p})}{n}}$

Para el análisis estadístico se utilizó el programa Stata versión 10.0 (College Station, Texas, USA). En la tabla 2 se presentan los datos epidemiológicos y las características operativas que se utilizaron en la evaluación económica.

Estructura del modelo. Se construyó un árbol de decisión (18-20) empleando TreeAge Pro 2009 (Williamstown, MA: TreeAge Software, Inc.) para simular los resultados en términos de los casos correctamente identificados por las pruebas rápidas y por el abordaje sindrómico, utilizando la prueba de Reacción en Cadena de la Polimerasa como patrón de oro (verdaderos positivos y verdaderos negativos) (figura 1).

En la simulación se practican pruebas rápidas a las pacientes o se realiza el abordaje sindrómico para el diagnóstico de la cervicitis por $C$. trachomatis. Cada alternativa puede tener un resultado positivo o negativo, y un número de verdaderos positivos y negativos según sus características operativas.

Supuestos del modelo. El principal supuesto de esta evaluación económica es que la cervicitis es causada con mayor frecuencia por la C. trachomatis, soportado en la prevalencia antes mencionada y en la baja prevalencia de infección por N. Gonorrhoeae $(1,4 \%$ (IC $95 \%$ 0,9-2,2) (12). El consenso de expertos determinó realizar la evaluación económica solamente para el diagnóstico de la cervicitis por C. trachomatis.

Análisis. Se calculó la razón de costo-efectividad incremental definida como el cociente entre la diferencia de costos y la diferencia de efectos (casos correctamente identificados). Se realizaron análisis de sensibilidad univariados y probabilísticos. Para el análisis de sensibilidad probabilístico se realizó una simulación de Montecarlo con 10.000 iteraciones; se utilizó la distribución de probabilidad uniforme

Tabla 1.

Costos empleados en la evaluación económica del diagnóstico etiológico basado en el uso de pruebas rápidas frente a diagnóstico sindrómico para la infección cervical por $C$. trachomatis

\begin{tabular}{|c|c|c|c|c|c|c|c|}
\hline \multirow{3}{*}{$\begin{array}{c}\text { Evento } \\
\text { generador } \\
\text { de costo }\end{array}$} & \multicolumn{3}{|c|}{$\begin{array}{l}\text { Análisis de sensibilidad } \\
\text { determinístico }\end{array}$} & \multicolumn{3}{|c|}{$\begin{array}{c}\text { Análisis de sensibilidad } \\
\text { probabilístico }\end{array}$} & \multirow{3}{*}{ Fuente } \\
\hline & \multirow{2}{*}{$\begin{array}{c}\text { Límite } \\
\text { inferior }^{\tau} \\
(\$)\end{array}$} & \multirow{2}{*}{$\begin{array}{c}\text { Caso } \\
\text { base }^{\tau \tau} \\
(\$)\end{array}$} & \multirow{2}{*}{$\begin{array}{c}\text { Límite } \\
\text { superior }^{\tau \tau \tau} \\
(\$)\end{array}$} & \multirow[b]{2}{*}{ Distribución } & \multicolumn{2}{|c|}{ Parámetros } & \\
\hline & & & & & $\alpha$ & $\lambda$ & \\
\hline Acon ${ }^{\circledR D u o}$ & 8.715 & 12.450 & 16.185 & Gamma & $81.340,0$ & 6,5 & Proveedor \\
\hline Acon ${ }^{\circledR P l a t e}$ & 4.742 & 6.775 & 8.807 & Gamma & $44.263,3$ & 6,5 & Proveedor \\
\hline QuickVue® & 12.600 & 18.000 & 20.800 & Gamma & $154.887,8$ & 8,6 & Proveedor \\
\hline $\begin{array}{c}\text { Consulta } \\
\text { ambulatoria de } \\
\text { medicina } \\
\text { especializada }\end{array}$ & 7.769 & 8.080 & 9.198 & Gamma & $179.092,2$ & 22,2 & $\begin{array}{l}\text { ISS }+25 \%^{\tau} \\
\text { ISS }+25 \%^{\tau \tau} \\
\text { ISS }+25 \%^{m}\end{array}$ \\
\hline
\end{tabular}


Figura 1.

Árbol de decisión empleado en la evaluación económica del diagnóstico etiológico basado en el uso de pruebas rápidas frente a diagnóstico sindrómico para la infección cervical por $C$. trachomatis

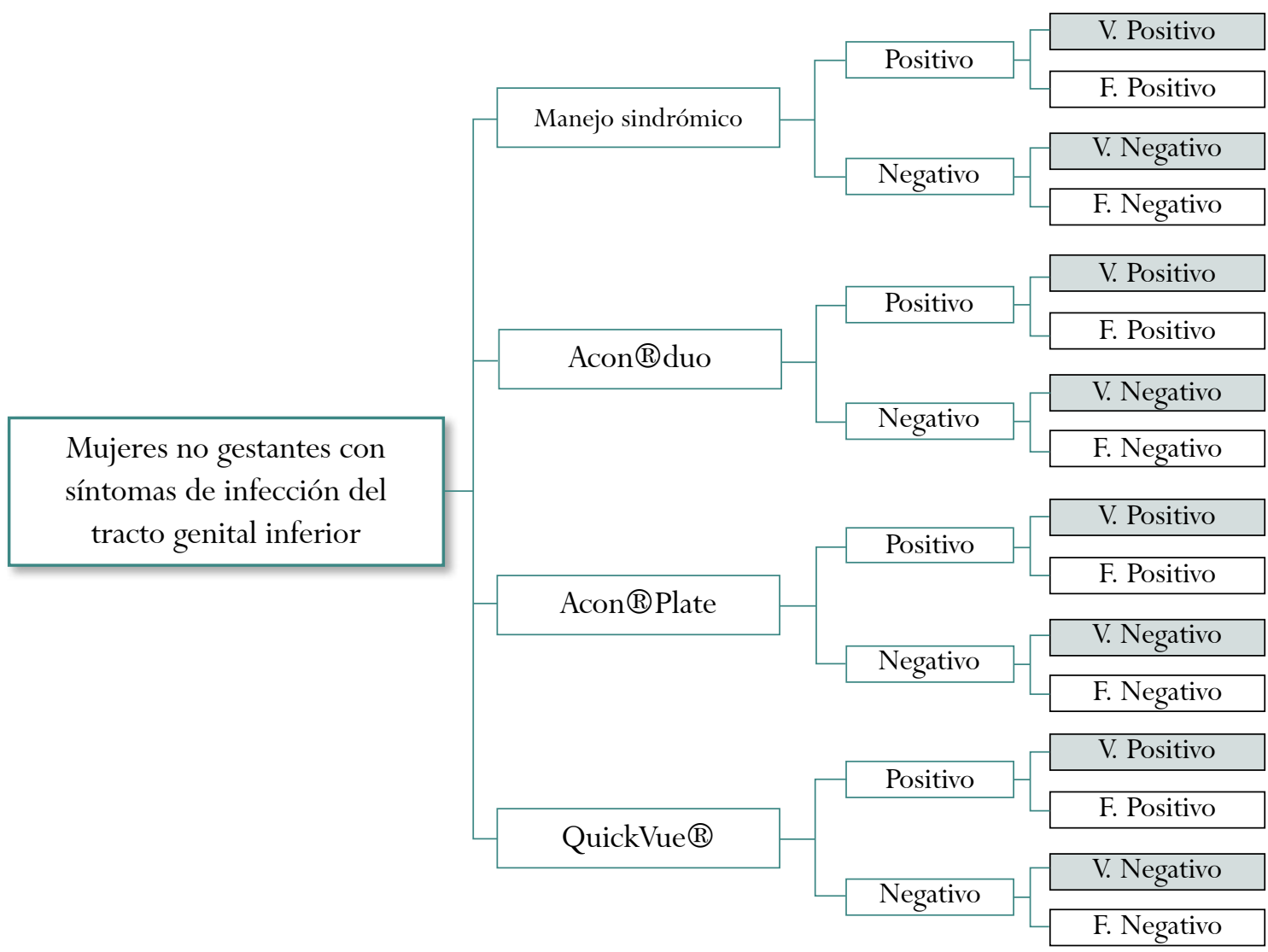

Fuente: elaboración de los autores.

para las características operativas de las pruebas y los datos epidemiológicos. A los costos se les asignó la distribución de probabilidad Gamma (21-23).

Aspectos éticos. El estudio base fue sometido al comité de ética de la Universidad Nacional. Todas las pacientes ingresadas firmaron el consentimiento informado.

\section{RESULTADOS}

Los resultados del modelo indican que la alternativa más costosa y más efectiva fue QuickVue ${ }^{\circledR}$, seguida de Acon®Plate y del abordaje sindrómico. Acon®Duo fue una estrategia dominada (figura 2). La razón de costo-efectividad incremental de QuickVue®, comparada con Acon®Plate, fue de $\$ 430.671$ y la de Acon $®$ Plate comparada con el abordaje sindrómico fue de $\$ 79.747$ (tabla 3).

\section{Manejo de la incertidumbre}

Análisis de sensibilidad univariado. El diagrama de tornado (figura 3) muestra que la razón de costoefectividad incremental es afectada principalmente por la prevalencia de $C$. trachomatis y por las características operativas de las pruebas.

Análisis de sensibilidad probabilístico. Los resultados de la simulación de Montecarlo se presentan en la figura 4.

En ausencia de un umbral para Colombia, a fin de determinar la costo-efectividad de las alternativas evaluadas, se asumieron posibles disponibilidades a pagar que se representan en el análisis por medio de la pendiente de las rectas (líneas negra, roja y azul). En el gráfico se observa que si la disponibilidad a pagar por un caso correctamente identificado adicional es \$200.000, QuickVue® sería la mejor 


\section{Tabla 2.}

Prevalencia y características operativas de las pruebas rápidas y diagnóstico sindrómico en la infección por $C$. trachomatis empleados en la evaluación económica

\begin{tabular}{|c|c|c|c|c|c|c|c|}
\hline \multirow{3}{*}{$\begin{array}{l}\text { Evento } \\
\text { generador } \\
\text { de costo }\end{array}$} & \multicolumn{3}{|c|}{$\begin{array}{c}\text { Análisis de sensibilidad } \\
\text { determinístico }\end{array}$} & \multicolumn{3}{|c|}{$\begin{array}{l}\text { Análisis de sensibilidad } \\
\text { probabilístico }\end{array}$} & \multirow{3}{*}{ Fuente } \\
\hline & \multirow{2}{*}{$\begin{array}{l}\text { Límite } \\
\text { inferior }\end{array}$} & \multirow{2}{*}{$\begin{array}{l}\text { Caso } \\
\text { base }\end{array}$} & \multirow{2}{*}{$\begin{array}{c}\text { Límite } \\
\text { superior }\end{array}$} & \multirow[b]{2}{*}{ Distribución } & \multicolumn{2}{|c|}{ Parámetros } & \\
\hline & & & & & $\alpha$ & B & \\
\hline $\begin{array}{l}\text { Sensibilidad } \\
\text { Acon }{ }^{\circledR D u o} \\
\text { C. trachomatis }\end{array}$ & 0,19 & 0,31 & 0,44 & Uniforme & 0,19 & 0,44 & \multirow{9}{*}{$\begin{array}{l}\text { Base de datos } \\
12\end{array}$} \\
\hline $\begin{array}{l}\text { Sensibilidad } \\
\text { Acon } ® \text { Plate } \\
\text { C. trachomatis }\end{array}$ & 0,08 & 0,23 & 0,45 & Uniforme & 0,08 & 0,45 & \\
\hline $\begin{array}{l}\text { Sensibilidad } \\
\text { Quickvue }{ }^{\circledR} \\
\text { C. trachomatis }\end{array}$ & 0,25 & 0,38 & 0,52 & Uniforme & 0,25 & 0,52 & \\
\hline $\begin{array}{l}\text { Sensibilidad } \\
\text { abordaje } \\
\text { sindrómico }\end{array}$ & 0,10 & 0,14 & 0,17 & Uniforme & 0,10 & 0,17 & \\
\hline $\begin{array}{l}\text { Especificidad } \\
\text { Acon }{ }^{\circledR} \text { Duo } \\
\text { C. trachomatis }\end{array}$ & 0,98 & 0,99 & 1,00 & Uniforme & 0,98 & 1,00 & \\
\hline $\begin{array}{l}\text { Especificidad } \\
\text { Acon }{ }^{\circledR P l a t e} \\
\text { C. trachomatis }\end{array}$ & 0,98 & 0,99 & 1,00 & Uniforme & 0,98 & 1,00 & \\
\hline $\begin{array}{l}\text { Especificidad } \\
\text { Quickvue }{ }^{\circledR} \\
\text { C. trachomatis }\end{array}$ & 0,98 & 0,99 & 1,00 & Uniforme & 0,98 & 1,00 & \\
\hline $\begin{array}{l}\text { Especificidad } \\
\text { abordaje } \\
\text { sindrómico }\end{array}$ & 0,88 & 0,91 & 0,93 & Uniforme & 0,88 & 0,93 & \\
\hline $\begin{array}{l}\text { Prevalencia de } \\
\text { C. trachomatis } \\
\text { con PCR }\end{array}$ & 0,08 & 0,10 & 0,11 & Uniforme & 0,08 & 0,11 & \\
\hline
\end{tabular}

\section{Tabla 3.}

Análisis de costo-efectividad del diagnóstico etiológico basado en pruebas rápidas frente a diagnóstico sindrómico en infección cervical por C. trachomatis

\begin{tabular}{|l|c|c|c|c|c|c|}
\hline Estrategia & $\begin{array}{c}\text { Costo } \\
(\$)\end{array}$ & $\begin{array}{c}\text { Costo } \\
\text { incremental } \\
(\$)\end{array}$ & Efectividad & $\begin{array}{c}\text { Efectividad } \\
\text { incremental }\end{array}$ & $\begin{array}{c}\text { Costo- } \\
\text { efectividad } \\
\text { promedio } \\
(\$)\end{array}$ & $\begin{array}{c}\text { Razón de costo- } \\
\text { efectividad } \\
\text { incremental }\end{array}$ \\
\hline Manejo sindrómico & 6.650 & & 0,833 & & 7.986 & \\
\hline Acon ${ }^{\circledR P l a t e}$ & 13.425 & 6.775 & 0,918 & 0,085 & 14.630 & 79.747 \\
\hline Acon ${ }^{\circledR D}$ Duo & 19.100 & 5.675 & 0,915 & $-0,003$ & 20.878 & (Dominado) \\
\hline QuickVue ${ }^{\circledR}$ & 24.650 & 11.225 & 0,944 & 0,026 & 26.120 & 430.671 \\
\hline
\end{tabular}


Figura 2.

Plano de costo-efectividad del diagnóstico etiológico basado en pruebas rápidas frente a diagnóstico sindrómico en infección cervical por C. trachomatis

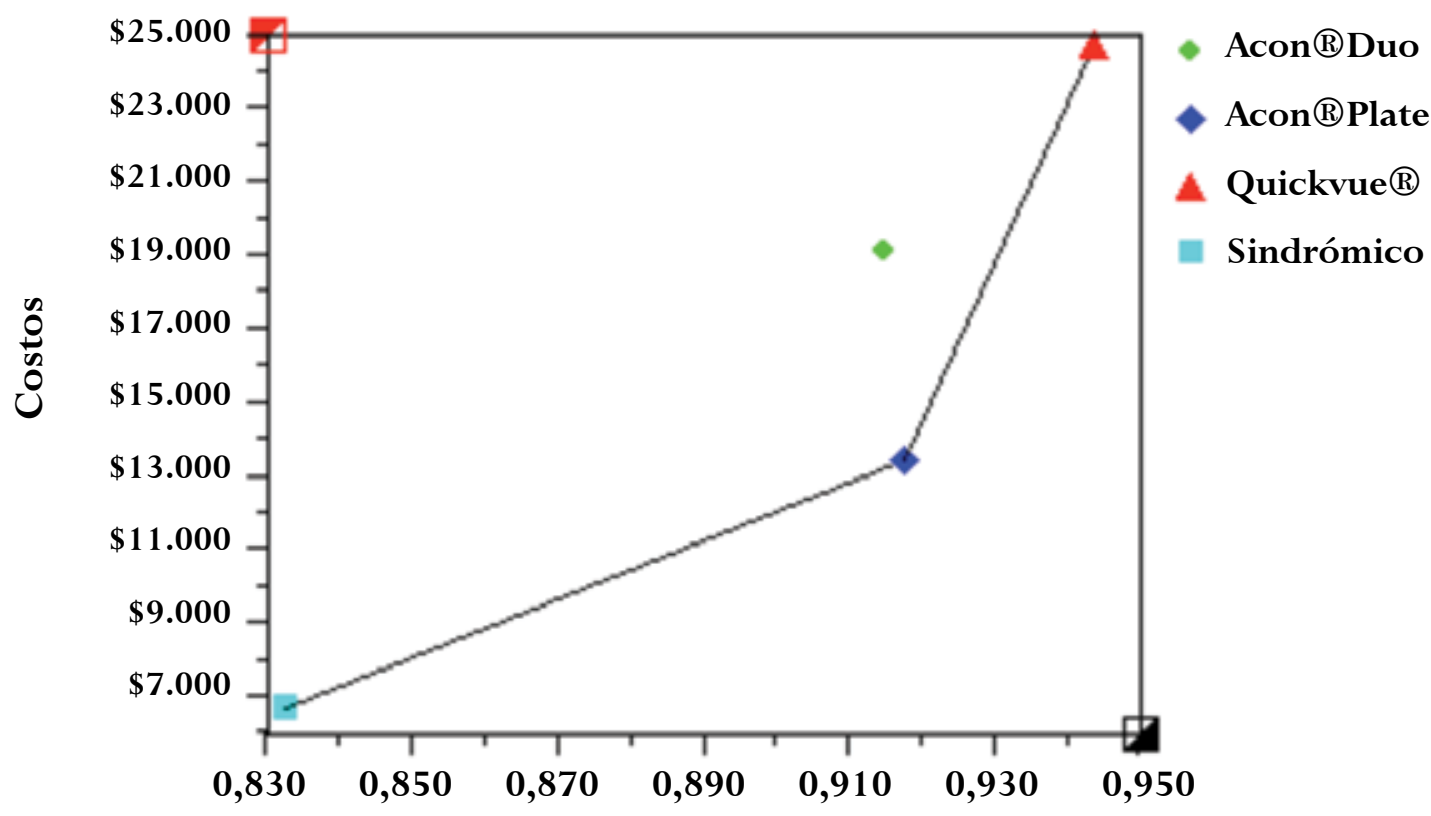

Efectividad

Figura 3.

Diagrama de Tornado en el análisis costo-efectividad del diagnóstico etiológico basado en pruebas rápidas frente a diagnóstico sindrómico en infección cervical por $C$. trachomatis

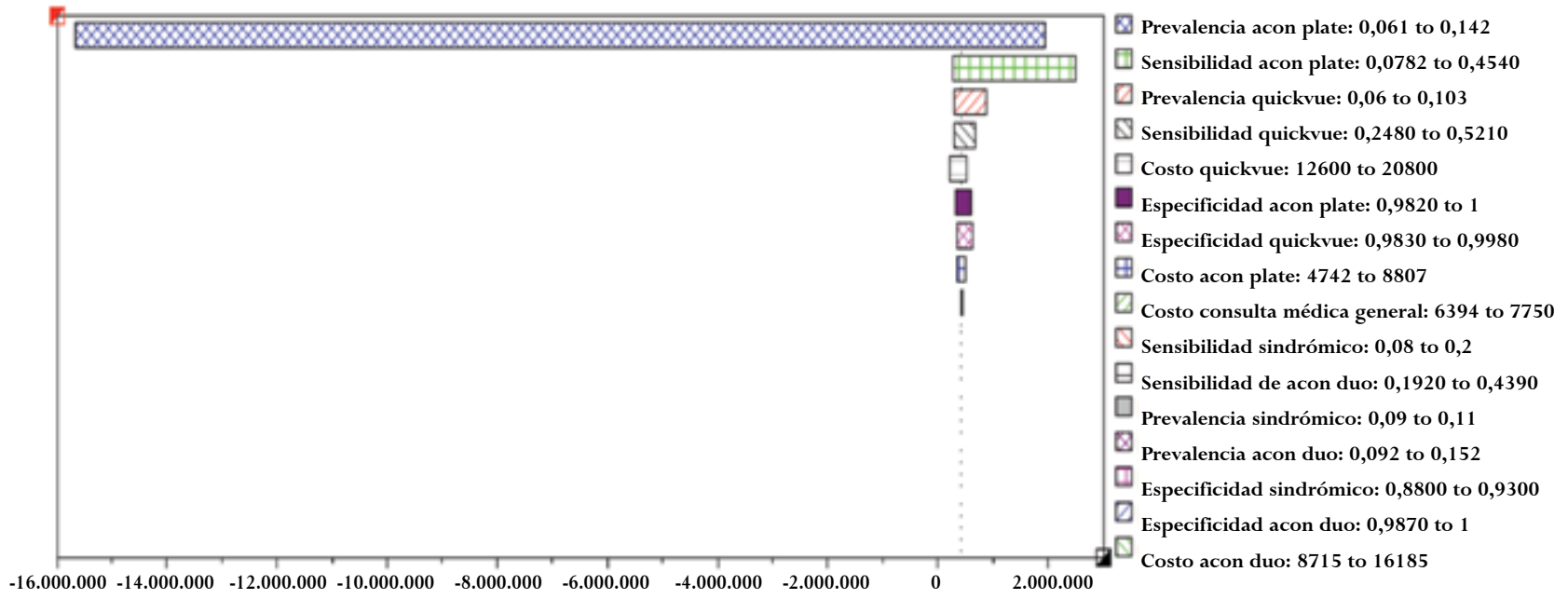

Razón de costo-efectividad 
Figura 4.

Diagrama de dispersión en el análisis costo-efectividad del diagnóstico etiológico basado en pruebas rápidas frente a diagnóstico sindrómico en infección cervical por C. trachomatis

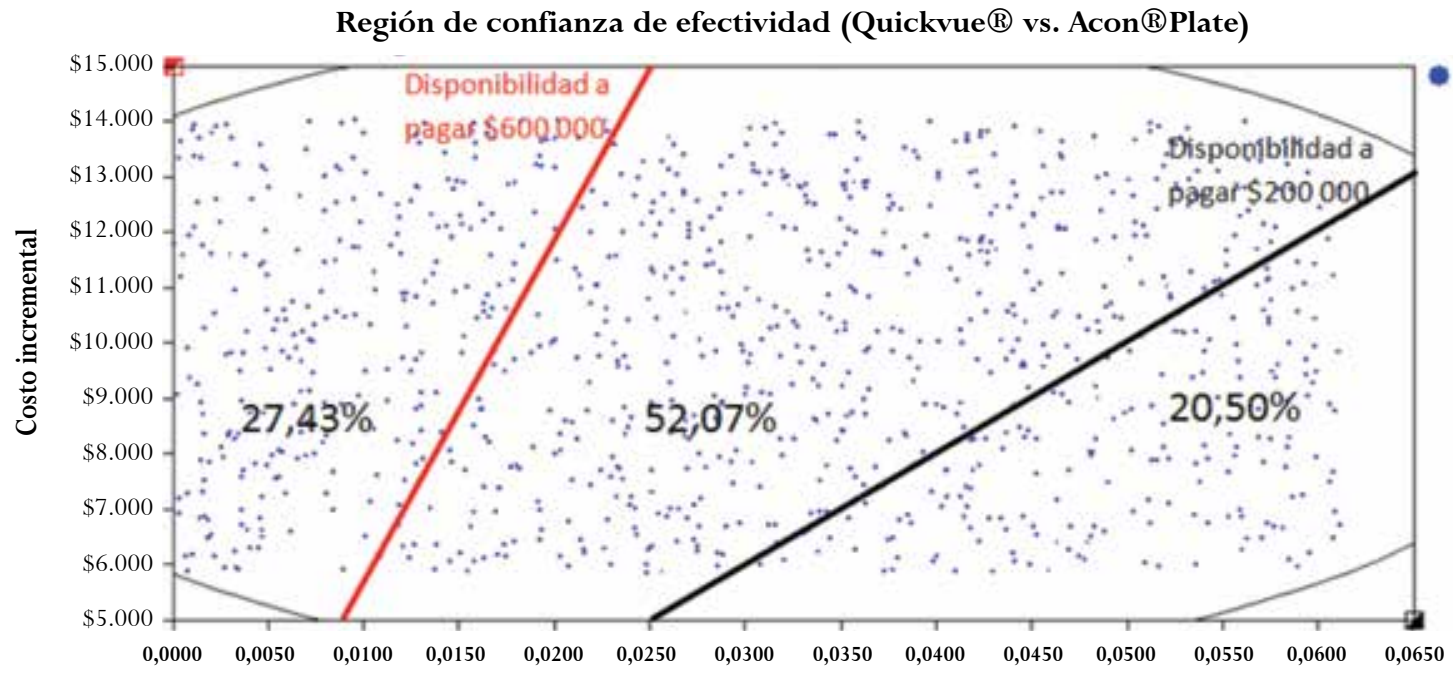

Efectividad incremental

alternativa en términos de costo-efectividad en el 20,5\% de los casos; para disponibilidades a pagar de $\$ 200.000$ y $\$ 600.000$, la costo-efectividad de esta alternativa estaría alrededor de 20,05 y 50,07\% de los casos, respectivamente. Finalmente, si la disponibilidad a pagar por un caso correctamente identificado es superior a \$600.000, QuickVue® sería costo-efectiva para el $100 \%$ de las iteraciones.

La curva de aceptabilidad (figura 5) muestra que el manejo sindrómico tiene mayor probabilidad de ser costo-efectivo para disponibilidades a pagar inferiores a \$ 200.000; para valores entre \$200.000 y

Figura 5.

Curva de aceptabilidad en la evaluación costo-efectividad del diagnóstico etiológico basado en pruebas rápidas frente a diagnóstico sindrómico en infección cervical por C. trachomatis

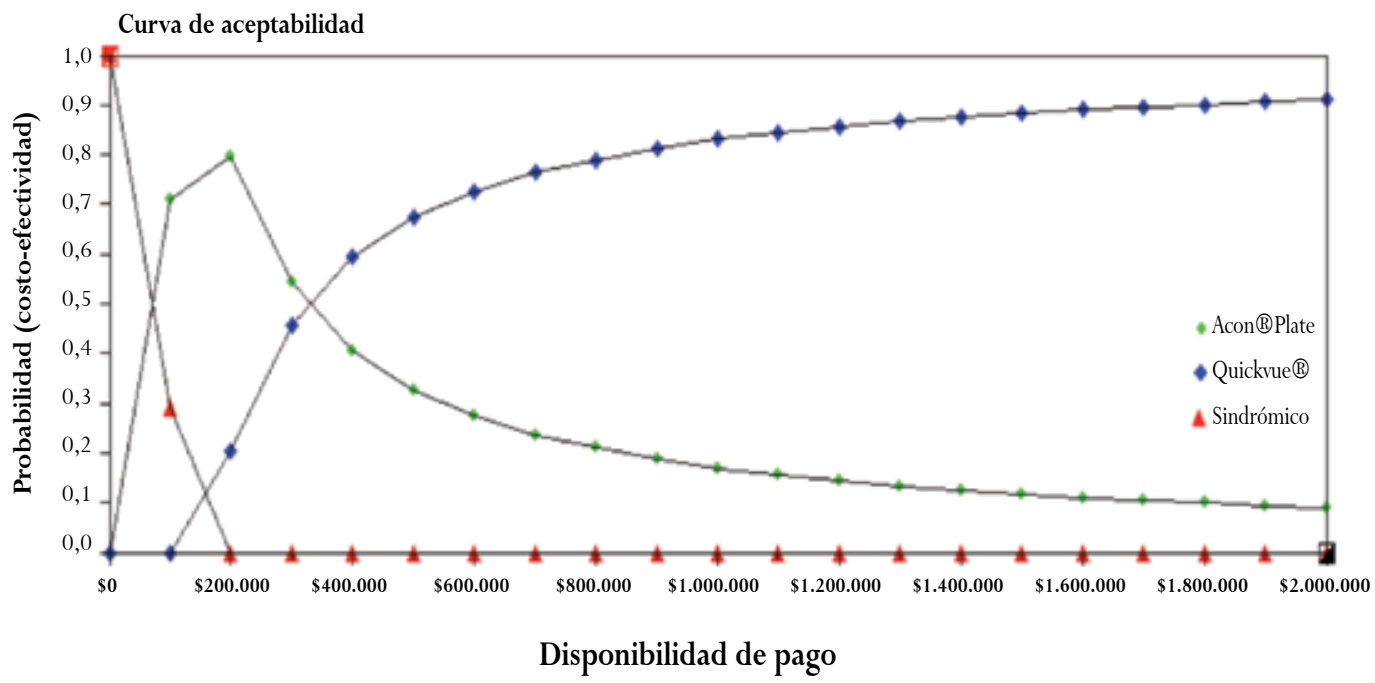


$\$ 320.000$, la alternativa con mayor probabilidad de ser costo-efectiva sería Acon®Plate, y para valores superiores a \$320.000, QuickVue® sería la mejor alternativa en términos de costo-efectividad.

\section{DISCUSIÓN}

Las pruebas rápidas son una tecnología más costosa pero más efectiva comparada con el abordaje sindrómico, que es la práctica clínica habitual en varios países de bajos o medianos ingresos $(24,25)$. Esta es la primera evaluación económica en el contexto colombiano diseñada para contribuir a la toma de decisiones acerca de la tecnología más apropiada para el diagnóstico de la cervicitis por $C$. trachomatis, teniendo en cuenta los recursos limitados y el impacto económico para el sistema de salud derivado de los costos y la exactitud de las aproximaciones diagnósticas $(26,27)$.

Es de anotar que durante el proceso de revisión de literatura de evaluaciones económicas no se encontraron estudios que compararan las alternativas diagnósticas que se consideraron en este trabajo. No obstante, los resultados de la evaluación económica son consistentes con los hallazgos de la revisión sistemática de Roberts et al. (28) sobre los métodos empleados para estimar la costo-efectividad de la tamización de C. trachomatis. De los 713 estudios identificados, los autores seleccionaron 59 para lectura completa, de los cuales 13 correspondieron a evaluaciones económicas de pruebas diagnósticas. El espectro de alternativas consideradas (pruebas de amplificación de ácido nucleico, reacción en cadena de la polimerasa, amplificación por desplazamiento de hebra, entre otras) dificulta determinar cuál de todas es la mejor en términos de costo-efectividad. Sin embargo, en general las pruebas para el diagnóstico de C. trachomatis mostraron ser costo-efectivas (28).

Esta evaluación económica tiene como fortaleza que se basa en un estudio que utiliza los patrones de oro diagnósticos aceptados para la condición de interés. El tamaño de la muestra del estudio fue apropiado de acuerdo con la prevalencia esperada de la cervicitis por $C$. trachomatis, y las característi- cas operativas de las pruebas fueron calculadas con validez y precisión. Adicionalmente, se contó con un bajo riesgo de mala clasificación y selección (12).

Una de las limitaciones de este estudio es que el principal supuesto de la evaluación económica acerca de la $C$. trachomatis como agente causal con mayor frecuencia de la cervicitis (12) puede no ser aceptado de manera global. No obstante, la simulación realizada tuvo en cuenta este aspecto y se realizaron análisis de sensibilidad tanto univariados como probabilísticos para mitigar la incertidumbre en relación con la prevalencia de la cervicitis por C. trachomatis.

Existen recomendaciones sobre emplear los años de vida ajustados por calidad (AVAC) como desenlace para las evaluaciones económicas desarrolladas en Colombia (15). En el presente análisis se utilizó como desenlace el número de casos correctamente diagnosticados, dado que el objetivo de las pruebas de diagnóstico es determinar si un paciente tiene o no tiene la infección (27). La sobrevida y calidad de vida dependen de otros factores que no se incorporaron en esta evaluación económica, tales como: la efectividad del tratamiento suministrado a la paciente después de conocer los resultados del diagnóstico, los eventos adversos que puedan ocurrir y las posibles complicaciones o secuelas (enfermedad pélvica inflamatoria o infertilidad) que se presentan habitualmente en un horizonte temporal más extenso.

De acuerdo con lo anterior, la razón de costoefectividad incremental por caso correctamente diagnosticado no es comparable con disponibilidades a pagar comprendidas entre 1 y 3 veces el producto interno bruto (PIB) per cápita, umbral que se recomienda cuando el desenlace son los AVAC (15). Por tanto, la costo-efectividad de las alternativas evaluadas es susceptible a la disponibilidad a pagar por un caso correctamente identificado adicional. La disponibilidad a pagar que fijen los tomadores de decisiones debería contemplar los resultados en salud derivados del diagnóstico correcto, los potenciales ahorros para el sistema de salud producto 
del tratamiento adecuado y del impacto financiero para una prevalencia estimada de cervicitis por $C$. trachomatis, de 9,7\% (12).

Para futuras evaluaciones económicas se recomienda considerar el impacto económico derivado de sobretratamientos o subtratamientos, y los costos de atender la ocurrencia de eventos como la infertilidad, que representan una carga económica para el sistema de salud teniendo en cuenta que los tratamientos son de alto costo y no son cubiertos por el Plan Obligatorio de Salud (POS).

\section{CONCLUSIÓN}

Para disponibilidades a pagar por un caso correctamente identificado adicional superiores a \$ 430.671 QuickVue ${ }^{\circledR}$ sería la alternativa costo-efectiva. Si la disponibilidad a pagar está entre \$79.747 y \$430.671, Acon®Plate sería la alternativa costoefectiva. Para disponibilidades a pagar inferiores a $\$ 79.747$, el abordaje sindrómico es costo-efectivo.

\section{FINANCIACIÓN}

Este trabajo fue financiado por Colciencias (Contrato 621 2009), la Universidad Nacional de Colombia, Sede Bogotá, y la Secretaría de Salud de Bogotá.

\section{REFERENCIAS}

1. World Health Organization. Sexually transmitted infections. Fact sheet. 2011 [Visitado 2014 ene 15]. Disponible en: http://www.who.int/mediacentre/ factsheets/fs $110 / \mathrm{en} /$.

2. Stamm WE. Chlamydia trachomatis infections in the adult. En: Holmes KK, Sparling PF, Stamm WE, et al. (eds.). Sexually transmitted diseases. 4th edition. New York: McGraw-Hill; 2008. p. 595-606.

3. Centers for Disease Control and Prevention. Gonorrhea-CDC Fact Sheet (Detailed Version). Atlanta, GA: U.S. Department of Health and Human Services, Centers for Disease Control and Prevention. 2014 [Visitado 2014 ene 15]. Disponible en: http:// www.cdc.gov/std/Gonorrhea/STDFact-gonorrheadetailed.htmThis\%20link\%20goes\%20offsite.
4. Valderrama J, Zacarias F, Mazin R. Maternal syphilis and congenital syphilis in Latin America: big problem, simple solution. Rev Panam Salud Pública. 2004: $16: 211-7$.

5. Organización Mundial de la Salud. Guías para el tratamiento de las infecciones de transmisión sexual. 2005 [Visitado 2014 ene 15]. Disponible en: http:// www.who.int/hiv/pub/sti/STIguidelines2003_es.pdf.

6. Ministerio de Salud y Protección Social de Colombia. Guía de Práctica Clínica para el abordaje sindrómico del diagnóstico y tratamiento de los pacientes con infecciones de transmisión sexual y otras infecciones del tracto genital. [Visitado 2014 oct 14]. Disponible en: http://gpc.minsalud.gov.co/Documents/GuiasPDF-Recursos/ITS/GPC_Prof_Sal_ITS.pdf .

7. Canadian Guidelines on Sexually Transmitted Infections. Ottawa: Public Health Agency of Canada. 2010 [Visitado 2014 ene 15]. Disponible en: http:// www.phac-aspc.gc.ca/std-mts/sti-its/cgsti-ldcits/ section-4-1-eng.php.

8. LeFevre ML. Screening for chlamydia and gonorrhea: U.S. Preventive Services Task Force recommendation statement. Ann Intern Med. 2014; 161:58-66 [Epub ahead of print].

9. Kamali A, Quigley M, Nakiyingi J, Kinsman J, KengeyaKayondo J, Gopal R, et al. Syndromic management of sexually-transmitted infections and behavior change interventions on transmission of HIV-1 in rural Uganda: a community randomised trial. Lancet. 2003;36:645-52.

10. Peeling RW, Holmes KK, Mabey D, Ronald A. Rapid tests for sexually transmitted infections (STIs): the way forward. Sex Transm Infect. 2006;82:v1-6.

11. Ministerio de Salud y Protección Social. Resolución 005521 (octubre 27 de 2013). [Visitado 2014 ene 15]. Disponible en: http://www.minsalud.gov. co/sites/rid/Lists/BibliotecaDigital/RIDE/DE/DIJ/ Resoluci\%C3\%B3n\%205521\%20de\%202013.pdf.

12. Angel-Müller E, Rodríguez A, Núñez-Forero LM, Moyano LF, González P, Osorio E, et al. Prevalencia y factores asociados a la infección por C. trachomatis, N. gonorrheae, T. vaginalis, C. albicans, Sífilis, VIH 
y vaginosis bacteriana en mujeres con síntomas de infección vaginal en tres sitios de atención de Bogotá, Colombia, 2010. Rev Colomb Obstet Ginecol. 2012; 63:14-24.

13. Tolosa JE, Rodríguez AE, Müller EA, Ruiz-Parra AI, Núñez-forero L, Moyano LF, et al. Accuracy of syndromic diagnosis and management of vaginal discharge and cervicitis in women of reproductive age in Bogotá, Colombia. Int J Gynecol Obstet. 2012;119:S3-S729.

14. Núñez-Forero L, Moyano LF, Angel-Müller E, Rodríguez A, Ruiz-Parra AI, Tolosa JE, et al. Diagnostic accuracy of rapid tests for C. Trachomatis, N. Gonorrhoeae and Syphilis at the point of care in women with symptoms of lower genital tract infection Int J Gynecol Obstet. 2012;119:S3-S350.

15. Instituto de Evaluación Tecnológica en Salud. Manual para la elaboración de evaluaciones económicas en salud. Bogotá D.C.: IETS. 2014 [Visitado 2014 ago 11]. Disponible en: http://www.iets.org.co/manuales/ Manuales/Manual\%20Evaluacion\%20economica.pdf.

16. Canadian Agency for Drugs and Technologies in Health. Guidelines for the economic evaluation of health technologies. 3rd ed. Ottawa: CADTH. 2006 [Visitado 2012 nov 10]. Disponible en: http://www. cadth.ca/en/products/health-technology-assessment/ publication/953.

17. Consejo Directivo del Instituto de Seguros Sociales. Acuerdo 256 de 2001, Por el cual se aprueba el "Manual de tarifas" de la Entidad Promotora de Salud del Seguro Social “EPS-ISS”. 2001 [Visitado 2012 ene 14]. Disponible en: http://lexsaludcolombia.files. wordpress.com/2010/10/tarifas-iss-2001.pdf.

18. Detsky AS, Naglie G, Krahn MD, Naimark D, Redelmeier DA. Primer on medical decision analysis: Part 1--Getting started. Med Decis Making. 1997;17:123-5.

19. Sánchez-Pedraza R, Gamboa O, Díaz JA. Modelos empleados para la toma de decisiones en el cuidado de la salud. Rev Salud Pública. 2008;10:178-88.

20. Detsky AS, Naglie G, Krahn MD, Redelmeier DA, Naimark D. Primer on medical decision analysis: Part 2--Building a tree. Med Decis Making. 1997;17: 126-35.

21. Briggs A, Claxton K, Sculpher M. Decision modelling for health economic evaluation. Oxford University Press; 2006.

22. Briggs A, Gray A. Handling uncertainty when performing economic evaluation of healthcare interventions. Health Technol Assess. 1999;3:1-134.

23. Briggs A. Handling Uncertainty in Cost-Effectiveness Models. Pharmacoeconomics. 2000;17:479-500.

24. Redwood-Campbell L, Plumb J. The syndromic approach to treatment of sexually transmitted diseases in low-income countries: issues, challenges, and future directions. J Obstet Gynaecol Can. 2002;24:417-24.

25. Trollope-Kumar K, Guyatt G. Syndromic approach for treatment of STIs: time for a change. Lancet. 2006;367:1380-1.

26. Laking G, Lord J, Fischer A. The economics of diagnosis. Health Econ. 2006;15:1109-20.

27. Sanghera S, Orlando R, Roberts T. Economic evaluations and diagnostic testing: an illustrative case study approach. Int J Technol Assess Health Care. 2013;29:53-60.

28. Roberts TE, Robinson S, Barton P, Bryan S, Low N. Screening for Chlamydia trachomatis: a systematic review of the economic evaluations and modelling. Sex Transm Infect. 2006;82:193-200. 